\title{
Comparação entre instrumentos de mensuração das consequências do trauma crânio-encefálico*
}

\author{
COMPARISONS AMONG MEASUREMENT TOOLS IN TRAUMATIC BRAIN INJURY OUTCOMES \\ COMPARACIÓN DE INSTRUMENTOS DE MEDIDA DE LAS CONSECUENCIAS \\ DEL TRAUMATISMO ENCÉFALOCRANEANO
}

\author{
Regina Márcia Cardoso de Sousa ${ }^{1}$
}

\begin{abstract}
RESUMO
O trauma crânio-encefálico (TCE) é considerado a causa mais importante de incapacidades entre jovens e a mais freqüente causa neurológica de morbidade. Em conseqüência, há um crescente interesse em instrumentos para monitorizar a recuperação após sua ocorrência. Nesse contexto, a Escala de Resultados de Glasgow (ERG) e a Disability Rating Scale (DRS) são instrumentos bastante utilizados. A atual investigação tem como objetivo comparar os resultados observados com o uso da DRS, ERG original e ampliada. Para atingir esse propósito, foram avaliadas as conseqüências póstrauma de 63 vítimas de TCE contuso, com idade entre 12 e 65 anos, em seguimento ambulatorial em um centro de trauma da cidade de São Paulo, entre seis meses e três anos após TCE. A comparação dos resultados permitiu concluir que, embora as três escalas fossem fortemente correlacionadas, a ERG ampliada destacou-se pela sua maior sensibilidade para detectar alterações nas vítimas com melhores condições pós-traumáticas.
\end{abstract}

\section{DESCRITORES}

Traumatismos cerebrais. Avaliação.

Escala de Resultados de Glasgow.

\section{ABSTRACT}

Traumatic brain injury (TBI) is considered the most important cause of disability among young people and the most common neurological cause of morbidity. Consequently, there is increasing interest in scales to monitor recovery in TBI. Among these scales, two have been widely adopted: the Glasgow Outcome Scale (GOS) and the Disability Rating Scale (DRS). The purpose of this paper is to compare the results found in DRS and in the original and extended GOS. Sixtythree closed-head injury victims, aged between 12 and 65, on an outpatient follow-up program at a trauma center in the city of São Paulo, with 6 months to 3 years post-TBI, had their characteristics and outcomes assessed. When comparing the results obtained by the scales, it was concluded that although the three of them were strongly correlated, extended GOS showed to be more sensitive in detecting changes in victims with better post-traumatic conditions.

\section{KEY WORDS \\ Brain injuries. \\ Evaluation. \\ Glasgow Outcomes Scale.}

\section{RESUMEN}

El traumatismo encéfalocraneano (TEC) es considerado la más importante causa de incapacidad entre jóvenes y la más frecuente de morbilidad neurológica. En consecuencia, hay un creciente interés en instrumentos para monitorear la recuperación después de ocurrido el TEC. En este contexto, la Escala de Resultados de Glasgow (ERG) y la Disability Rating Scale (DRS) son instrumentos bastante utilizados. La actual investigación tiene como objetivo comparar los resultados observados con el uso de la DRS, ERG original y ampliada. Para alcanzar ese propósito, fueron evaluadas las consecuencias post trauma de 63 víctimas de TEC, con edad entre 12 y 65 años, en seguimiento ambulatorio en un centro de trauma de la ciudad de Sao Paulo, entre seis meses y tres años después del TEC. La comparación de los resultados permitió concluir que, no obstante que las tres escalas estén fuertemente correlacionadas, la ERG ampliada se destacó por su mayor sensibilidad para detectar alteraciones en las víctimas con mejores condiciones post-traumáticas.

\section{DESCRIPTORES}

Traumatismos cerebrales. Evaluación.

Escala de Resultados de Glasgow.

\footnotetext{
* A Pesquisa contou com auxílio da Fundação de Amparo à Pesquisa do Estado de São Paulo (FAPESP), Processo no 01/09645-7

1 Professora Associada do Departamento de Enfermagem Médico-Cirúrgica da Escola de Enfermagem da Universidade de São Paulo(EEUSP) vian@usp.br.
} 


\section{INTRODUÇÃO}

As estatísticas brasileiras revelam que as Causas Externas estão entre as quatro mais freqüentes causas de mortalidade no País. Se excluídas as mortes por causa mal definidas, passam, então, a ocupar o segundo ou terceiro lugar ${ }^{(1)}$. No entanto, as conseqüências do trauma não se resumem nas mortes, muitas vítimas sobreviventes permanecem com deficiências, por longo tempo, ou então, seqüelas físicas e cognitivas permanentes.

No conjunto de lesões das causas externas, o Trauma Crânio-Encefálico (TCE) destaca-se em termos de magnitude e sobretudo como causa de morte e incapacidade. É a causa mais importante de morte e incapacidade entre jovens, e a mais freqüente causa neurológica de mortalidade e morbidade nos Estados Unidos ${ }^{(2)}$.

A Glasgow Outcome Scale, conhecida em nosso meio como Escala de Resultados de Glasgow (ERG), e a Disability Rating Scale (DRS) têm sido recomendadas para avaliar a capacidade funcional global em estudos clínicos de vítimas de TCE grave, mas podem também ser utilizadas em grupos com TCE de moderada gravidade ${ }^{(3)}$.

A ERG original é um dos instrumentos de medida da capacidade funcional mais comumente utilizados nas pesquisas sobre TCE. Foi publicada em $1975^{(4)}$ e contém cinco categorias para classificação dos pacientes: boa recuperação; incapacidade moderada; incapacidade grave; estado vegetativo persistente e morte. Sua principal qualidade está na facilidade de sua aplicação e a mais freqüente crítica está na grande amplitude de cada uma de suas categorias que não discrimina importantes diferenças clínicas, classificando, igualmente, indivíduos que apresentam condições funcionais muito distintas ${ }^{(5-12)}$.

Esta limitação foi reconhecida pelos próprios autores que sugeriram uma subdivisão da escala original para permitir uma maior sensibilidade na avaliação de pacientes que retornam à consciência ${ }^{(5)}$.

Desta forma, dentro das categorias originais dos indivíduos conscientes (boa recuperação, incapacidade moderada e incapacidade grave), foi proposta uma subdivisão dos indivíduos com melhor ou pior nível de recuperação. A escala ampliada passou, então, a apresentar oito categorias e seis de suas graduações foram utilizadas para designar a condição do indivíduo que retornou à consciência, após o trauma.

A DRS é também bastante utilizada como instrumento de mensuração da capacidade funcional após TCE, sendo elaborada para oferecer um método de medida sensível, confiável, válido, fácil de ser administrado desde a fase de coma da vítima de TCE até o retorno à comunidade ${ }^{(6,9)}$.
A DRS consiste de oito itens de avaliação que são incluídos em quatro indicadores: despertar, consciência e responsividade; habilidade cognitiva para atividades de autocuidado; dependência de terceiros e capacidade de adaptação psicossocial.

A pontuação atribuída ao indicador despertar, consciência e responsividade varia de 0 (zero) a 12 (doze); em relação à habilidade cognitiva para atividades de autocuidado, o escore atribuído é 0 (zero) a 9 (nove). A pontuação no indicador dependência de terceiros é 0 (zero) a 5 (cinco) e capacidade de adaptação psicossocial de 0 (zero) a 3 (três). A soma total dos escores máximos dos 4 indicadores é 29 pontos, entretanto, é prevista a pontuação 30 , atribuída às vítimas que morrem.

Na DRS, o escore total individual obtido classifica as vítimas em dez diferentes categorias. Assim, escore de 0 (zero) indica nenhum nível de incapacidade; de 1 (um), leve; de 2 (dois) ou 3 (três), parcial; de 4 (quatro) a 6 (seis), moderada; de 7 (sete) a 11 (onze), moderadamente grave; de 12 (doze) a 16 (dezesseis), grave; de 17 (dezessete) a 21 (vinte e um), extremamente grave; de 22 (vinte e dois) a 24 (vinte e quatro), estado vegetativo; de 25 (vinte e cinco) a 29 (vinte e nove), estado vegetativo extremo e pontuação de 30 (trinta) inclui os mortos.

Intuitivamente, a DRS por ser uma escala com variação na pontuação de 0 a 30, que resulta em 10 categorias, parece ser mais sensível que a ERG original. Investigações realizadas confirmaram a maior sensibilidade da DRS em relação à ERG original ${ }^{(6,8)}$.

Paradoxalmente, estudos mais recentes vêm se contrapondo a esses resultados, mostrando que o uso da DRS não oferece maior sensibilidade do que a ERG original e, até mesmo, do que a ERG simplificada nas categorias: dependentes e independentes ${ }^{(7,11,13)}$.

Frente a essas observações contraditórias, optou-se no presente estudo por comparar os resultados obtidos na avaliação da recuperação pós-traumática por meio da DRS, ERG original e ampliada, tendo em vista um grupo de vítimas em acompanhamento ambulatorial após TCE.

\section{MÉTODO}

O presente estudo foi realizado no ambulatório de hospital governamental, centro de referência para atendimento de vítimas de trauma na região oeste da Grande São Paulo.

Foram alvo desta investigação as vítimas de TCE contuso, atendidas no Pronto-Socorro desse hospital, nas primeiras 72 horas após evento traumático, que necessitaram de internação hospitalar e, posteriormente, mantiveram consultas agendadas no ambulatório de TCE para continuidade do tratamento. 
Foram também critérios de inclusão no estudo: a idade das vítimas, entre 12 e 65 anos; o comparecimento à consulta agendada, entre seis meses e três anos, após TCE; e o consentimento em participar do estudo.

A exclusão de vítimas menores de 12 e maiores de 65 anos visou minimizar a influência da idade nos resultados relacionados à capacidade funcional. Nesse aspecto, vale ressaltar que a recuperação pós-traumática é um processo dinâmico, dependente do tempo que interfere nas conseqüências observadas, portanto, o período mínimo de seis meses e máximo de três anos foram determinados para as avaliações após o trauma ${ }^{(14-15)}$.

Os dados referentes às vítimas foram obtidos por intermédio da consulta dos prontuários e em entrevistas realizadas no ambulatório de TCE, antes dos atendimentos médicos.

A busca de informações no prontuário permitiu a seleção das vítimas para a entrevista, uma vez que os registros possibilitaram averiguar se a vítima atendia aos critérios de inclusão pré-estabelecidos. Após a seleção, as pessoas que se adequaram à pesquisa, tiveram seus prontuários criteriosamente analisados e deles extraídos os dados necessários para caracterização do grupo estudado.

Como instrumento de medida de conseqüências após TCE, foram utilizadas as escalas, DRS, ERG original e ampliada. A aplicação concomitante dos três instrumentos durante as entrevistas com vítimas e familiares permitiu a comparação dos resultados obtidos, por meio das pontuações obtidas e classificação das vítimas em categorias.

Para determinar a pontuação parcial e total da DRS, utilizou-se o formulário e a instrução proposta pelos autores da escala $^{(6)}$, apresentados no Anexo A. O escore da ERG ampliada foi estabelecido, utilizando-se os critérios de pontuação anteriormente publicados ${ }^{(14-15)}$ e mostrados no Anexo B.

Depois de determinado o escore da ERG ampliada, estabeleceu-se a pontuação da ERG original. A transformação direta da pontuação da ERG ampliada para original é facilitada à medida que as categorias adicionadas são subdivisão de uma das categorias da ERG em sua versão original.

Ao término da coleta, os dados foram armazenados em banco de dados computadorizado, os resultados foram organizados para serem aqui apresentados em freqüências absolutas e relativas e ilustrados na forma de tabelas e figuras.

Para certificação da relação existente entre as categorias da DRS, ERG original e ampliada, foi aplicada a prova estatística do Coeficiente de Correlação por postos de Spearman. Essa prova estatística foi feita admitindo-se erro de primeira espécie de $5 \%$.

\section{RESULTADOS}

Entre junho de 1999 e março de 2000, foram avaliadas 63 vítimas, em acompanhamento ambulatorial que tiveram lesão contusa e atenderam aos demais critérios de inclusão estabelecidos para este estudo. A análise dessas vítimas, com idade de 12 a 65 anos, mostrou as seguintes características demográficas da casuística estudada: idade média de 33,8 anos, desvio padrão de 14,2 ; a grande maioria $(81,0 \%)$ era do sexo masculino; uma proporção similar de casados e solteiros; a escolaridade média de 5,3 anos e 71,4\% das vítimas com ocupação remunerada no período que antecedeu o trauma.

Quanto às características clínicas, a maioria, 58,7\%, era vítima de acidente de transporte, $44,4 \%$ tinham presença de algum dos seguintes antecedentes: abuso de uso de álcool ou drogas, trauma anterior, epilepsia ou distúrbio psiquiátrico. Além disso, 52,4\% das vítimas apresentaram complicações pós-traumáticas e mais da metade superou dez dias de internação na fase aguda de tratamento, média de 18,8 dias e desvio padrão 21,9. O Injury Severity Score (ISS) foi em média de 20,2 $( \pm 8,0)$ e diferiu na grande maioria das vítimas do New Injury Severity Score (NISS) que apresentou média de 31,8 $( \pm 13,6)$. Em relação à gravidade do TCE, $77,8 \%$ das vítimas tinha o máximo Abbreviated Injury Scale (AIS) da região cabeça de 4 (lesão grave) ou 5 (lesão crítica), porém, somente 41,7\% apresentou indicação de TCE grave, quando a Escala de Coma de Glasgow foi o instrumento de medida utilizado, ou seja, obteve escore inferior a nove nessa escala.

Tabela $1 \ddagger$ Classificação das vítimas ( $\mathrm{n}=63$ ), segundo ERG original, ampliada e DRS -1999-2000 - São Paulo

ERG (ORIGINAL)

Boa recuperação

Incapacidade moderada

Incapacidade grave

Estado vegetativo persistente

ERG (AMPLIADA)

Recuperação total

Boa recuperação

Incapacidade moderada

Incapacidade moderada acentuada

Incapacidade grave

Incapacidade grave acentuada

Estado vegetativo persistente

\section{DRS}

Nenhuma (0)

$25(39,7 \%)$

Leve (1)

Parcial (2-3)

$16(25,4 \%)$

$05(7,9 \%)$

Moderada (4-6)

$13(20,6 \%)$

Moderadamente grave (7-11)

$03(4,8 \%)$

Grave (12-16)

$01(1,6 \%)$

Extremamente grave (17-21)

Estado vegetativo (22-24)

Estado extremamene vegetativo (25-29)
$06(9,5 \%)$

$-\cdot$

$15(23,8 \%)$

$06(9,5 \%)$ tame 
Conforme se observa na Tabela 1, a maioria do grupo apresentou, no período entre seis meses e três anos, nenhuma ou leve incapacidade, segundo a DRS, boa recuperação, segunda a ERG original e sendo assim, obteve total ou boa recuperação, conforme a ERG ampliada. No geral, as vítimas concentraram-se nas categorias das escalas que indicavam melhores resultados, sendo poucas as indicações de dependência acentuada pós-trauma.

Segundo a DRS, as vítimas distribuíram-se em 6 das 9 categorias propostas para sobreviventes. Indivíduos em condição extremamente grave, assim como em estado vegetativo não foram identificados.

Tabela 2 - Correlação entre os escores da ERG original, ampliada e DRS - São Paulo - 1999-2000

\begin{tabular}{lcc}
\hline \multicolumn{1}{c}{ Escalas } & $\begin{array}{c}\text { Coeficiente de } \\
\text { Correlação }\end{array}$ & P \\
\hline ERG ampliada x DRS & 0,89 & $<0,001$ \\
ERG original x DRS & 0,89 & $<0,001$ \\
\hline
\end{tabular}

Os dados contidos na Tabela 2 acusam uma forte correlação, estatisticamente significativa entre a DRS e a ERG original e ampliada, sugerindo equivalência nos resultados das escalas. Desta forma, quanto mais acentuada a incapacidade indicada nos escores da DRS, piores também foram as conseqüências pós-traumáticas indicadas pela ERG original e ampliada.

Para uma análise mais detalhada da relação entre as escalas, são apresentadas a média e a variação da pontuação obtida na DRS em cada categoria da ERG original, na Figura 1 e da ERG ampliada na Figura 2.

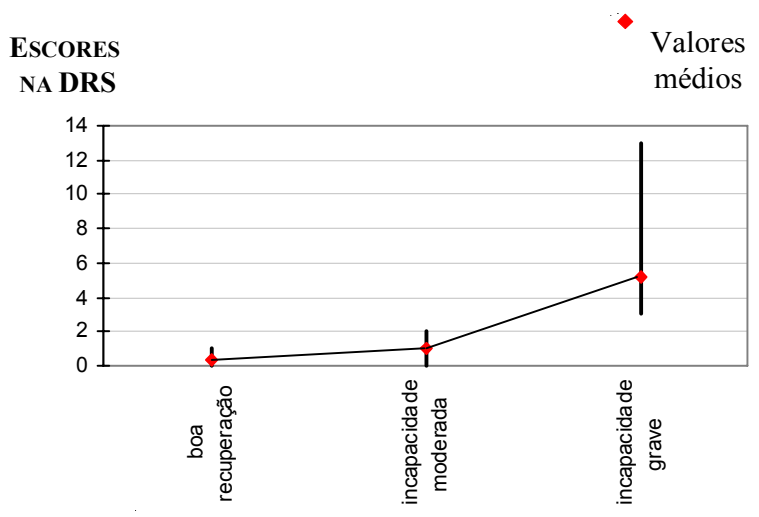

Categorias da ERG ORIGINAL

Figura 1 - Média e variação dos escores da DRS para as categorias da ERG original - São Paulo -1999-2000
Na Figura 1, observa-se que os valores médios da DRS foram progressivamente maiores à medida que piores resultados foram apresentados pelas vítimas, segundo a ERG original. Na categoria boa recuperação, as vítimas apresentaram valor médio na DRS de 0,3 , variação na pontuação de 0 a 1 , com 70,6\%, com escore 0 (zero). As vítimas com incapacidade moderada mostraram média de 1,0 , pontuação de 0 (zero) a 2, com grande maioria, $75,0 \%$, com escore 1 . A sobreposição na variação dos escores da DRS foi observada quando essas duas categorias da ERG original foram analisadas, entretanto, vítimas com incapacidade grave, apresentaram escores diferenciados na DRS. Para elas, o escore médio nessa escala foi 5,1 com variação de 3 a 13 . Tais resultados indicam que as principais diferenças entre as duas escalas encontram-se nas categorias que apontam os melhores resultados, isto é, conseqüências pós-traumáticas de menor gravidade.

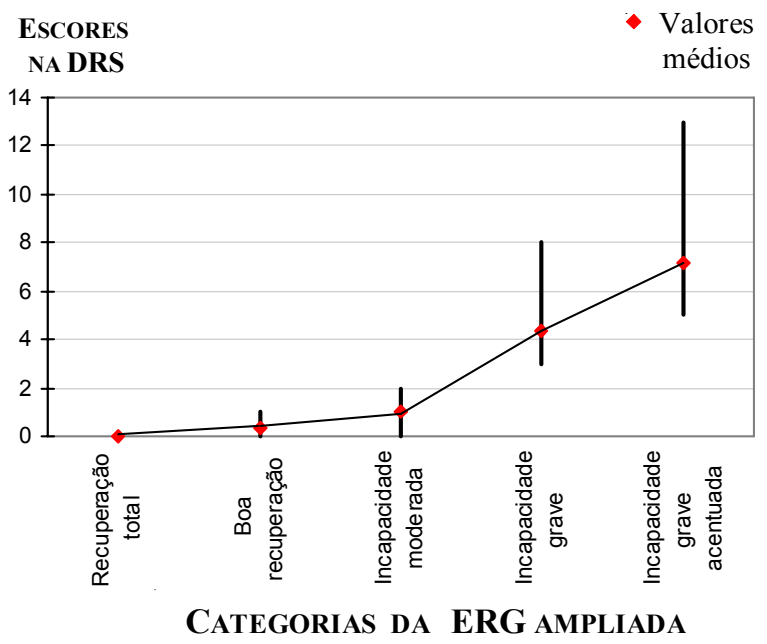

Figura 2 - Média e variação do escore da DRS para as categorias da ERG ampliada - São Paulo -1999-2000

A Figura 2 também mostra valores médios crescentes da DRS conforme as categorias de maior gravidade na ERG ampliada. Esta Figura evidencia que vítimas com recuperação total têm DRS igual a 0 (zero), além de mostrar a diferença nos escores da DRS, quando vítimas com incapacidade grave são comparadas às de incapacidade grave acentuada. O valor médio para o grupo com incapacidade grave foi 4,3 , a variação de 3 a 8 e a mediana 4 . Por outro lado, no grupo com incapacidade grave acentuada, a média foi 7,2, a variação de 5 a 13 e a mediana 6,5. A sobreposição na variação dos escores da DRS foi observada em $40,0 \%$ das vítimas com incapacidade grave que apresentaram escore igual ou maior que 5 . 
Tabela 3 - Distribuição das vítimas, segundo indicação de alterações pós-traumáticas com o uso da DRS e ERG original e ampliada - 1999-2000 - São Paulo

\begin{tabular}{lcccrcr}
\hline \multicolumn{1}{c}{ Escalas } & & DRS & \multicolumn{2}{c}{ ERG-Ampliada } & \multicolumn{2}{c}{ ERG-Original } \\
\hline \multicolumn{1}{c}{ Alteração Pós-Traumática } & $\mathbf{n}$ & $\mathbf{\%}$ & $\mathbf{n}$ & $\mathbf{\%}$ & $\mathbf{n}$ & $\%$ \\
\hline Indicadas & 38 & 60,3 & 57 & 90,5 & 28 & 44,4 \\
Não indicadas & 25 & 39,7 & 06 & 9,5 & 35 & 55,6 \\
\hline Total & 63 & 100,0 & 63 & 100,0 & 63 & 100,0 \\
\hline
\end{tabular}

Os dados da Tabela 3 permitem a comparação entre DRS, ERG original e ampliada, quanto à detecção da presença de alterações pós-traumáticas. Para tanto, as categorias das escalas que indicam a menor conseqüência pós-traumática, nenhuma incapacidade na DRS, recuperação total na ERG ampliada e boa recuperação na ERG original foram equiparadas à indicação de ausência de alteração na fase pós-traumática e todas as demais categorias das escalas foram consideradas indicação de presença dessa alteração.

Os resultados da Tabela 3 mostram que a ERG ampliada seguida pela DRS, apontaram em maiores freqüências alterações pós-traumáticas nas vítimas. A ERG original detectou menos da metade e a DRS cerca de um terço das alterações apontadas pela ERG ampliada.

\section{DISCUSSÃO}

De forma similar ao atual, outros estudos também têm mostrado que mesmo as vítimas de TCE grave apresentam, em sua maioria, boa recuperação na ERG original, no período de seis meses a um ano, após trauma ${ }^{(14-19)}$. No entanto, cabe lembrar que a inclusão das vítimas nessa categoria da escala não implica ausência de conseqüências pós-trauma.

Observa-se que nos resultados da ERG ampliada, apresentados na Tabela 1, que das 34 vítimas incluídas na categoria boa recuperação da escala original, somente 6 apresentaram recuperação total, permanecendo em todas as demais alterações cognitivas, físicas ou comportamentais.

A maior sensibilidade da ERG ampliada em relação à ERG original permitiu ainda distinguir nas vítimas categorizadas com incapacidade grave, aquelas que necessitavam de auxílio de outrem para as atividades básicas de autocuidado daquelas com menor dependência. Vítimas extremamente dependentes, em estado vegetativo persistente, não foram identificadas. As características e o tempo de sobrevida após trauma dessas vítimas tornam pouco viável sua participação em grupos de acompanhamento ambulatorial. Também entre as vítimas com incapacidade moderada não foram observadas pessoas independentes que fizessem uso de artefatos ou adaptações para executarem suas atividades diárias, isto é, indivíduos com incapacidade moderada acentuada.
Assim como em outros grupos de trauma e TCE, na amostra estudada observou-se que a maioria das vítimas $(66,7 \%)$ alcançou na ERG ampliada recuperação condizente com a vida independente (boa ou total recuperação ou incapacidade moderada) e as gravemente incapacitadas, acentuadamente dependentes, foram em pequena proporção $(9,5 \%)^{(14-17,20)}$.

Estudos que aplicaram a DRS em vítimas de TCE, apresentaram perfil similar ao aqui observado e mostraram as vítimas distribuídas somente nas categorias: nenhuma, leve, parcial, moderada, moderadamente grave e grave incapaci$\operatorname{dade}^{(7,13,21)}$. Os piores resultados com o uso dessa escala foram observados em pessoas internadas em serviços de reabilitação. Nesse grupo de vítimas, o escore médio na admissão foi 13 (treze), a máxima pontuação observada no presente estudo, e na alta 6 (seis), valor alcançado pela minoria dos aqui estudados ${ }^{(22)}$.

Frente ao grupo de vítimas analisado, a comparação entre os resultados obtidos pela DRS, ERG original e ampliada, permitiu concluir que, embora os resultados das três escalas sejam fortemente correlacionados, a ERG ampliada, destacou-se pela sua maior sensibilidade para detectar alterações nas vítimas com melhores condições pós-traumáticas.

Conforme mencionado na introdução do presente estudo, a ERG ampliada foi uma proposta para melhorar a sensibilidade da ERG original, apresentada em $1981^{(5)}$. Amplia para 8 as 5 categorias da ERG original, porém, essa modificação não foi acompanhada de critérios claros para sua aplicação. Limites não foram determinados para possibilitar ao avaliador discernir com segurança um indivíduo com uma recuperação total de alguém com boa recuperação. De forma semelhante, essa imprecisão ficou mantida também na subdivisão das categorias incapacidade moderada e grave da ERG original.

Provavelmente, a subjetividade da proposta de modificação desses autores, descrita como uma subdivisão em melhor e pior de três categorias da escala, não estimulou sua utilização e provocou pouca credibilidade dentro da comunidade científica. Assim, poucas foram as investigações que fizeram uso da ERG ampliada nesses últimos 20 anos, e a ERG original, ainda que criticada, mantém-se como um dos instrumentos mais usados para avaliar a capacidade funcional após $\mathrm{TCE}^{(5)}$. 
Pesquisadores holandeses ${ }^{(23)}$ publicaram estudo estabelecendo critérios para as diferentes categorias da ERG ampliada. Analisaram a partir, de então, a confiabilidade das duas versões da ERG e mostraram um valor bem mais elevado do coeficiente Kappa, quando a escala de 5 categorias foi utilizada. Nesse estudo, uma considerável discordância entre avaliadores, também, foi observada na ERG original, trazendo à tona outra crítica a essa escala: a ausência de uma entrevista estruturada que direcione os aspectos relevantes a serem considerados na avaliação realizada.

No ano de 1993, a autora do presente estudo frente ao desafio de estudar o padrão de recuperação das vítimas de $\mathrm{TCE}^{(14-15)}$, elaborou formulário direcionando a entrevista realizada e fixou critérios para aplicação da ERG ampliada, conforme descrito em outras publicações ${ }^{(14-15)}$. Nessa tarefa, o documento Classificação Internacional das Deficiências, Incapacidades e Desvantagens (handicaps), publicado pela Organização Mundial da Saúde ${ }^{(24)}$, foi a principal diretriz na determinação das atividades a serem avaliadas e, também, auxiliou na tarefa de estabelecer os critérios de inclusão das vítimas nas categorias da escala.

Recentemente, autores ${ }^{(25)}$ adotaram um formato-padrão para entrevista utilizada na avaliação de resultados, fornecendo também um guia para aplicação da ERG em suas duas versões. Entretanto, no presente estudo, a proposta anteriormente elaborada foi mantida, tendo em vista a experiência satisfatória já observada.

No entanto, vale aqui explicitar que as diferenças entre as duas propostas concentram-se na forma de diferenciação entre vítimas com incapacidade moderada e incapacidade moderada agravada, assim como entre aquelas com incapacidade grave e incapacidade grave acentuada. Em nossa proposta, a diferenciação das vítimas com incapacidade moderada foi pautada na necessidade de dispositivos ou ambiente adaptado para manutenção da independência, enquanto na outra proposta ${ }^{(25)}$ a diferença está na freqüência das dificuldades nos relacionamentos sociais, na restrição das atividades sociais e de lazer e na capacidade de traba1ho. Quanto à incapacidade grave, essa proposta estabelece que os pacientes devem ser incluídos na mais elevada categoria, quando não podem permanecer sozinhos em sua residência por oito horas. Em nossos estudos, a capacidade de manter as atividades de banhar-se, alimentar-se, deslocarse dentro do local que se encontra, higiene em relação à função excretora e arrumar-se foi a base para diferenciar as duas categorias dos indivíduos com incapacidade grave.

Correlações equivalentes às observadas na Tabela 2, 0,89, foram observadas ${ }^{(26)}$, quando confrontaram a DRS com a ERG original e ampliada e utilizaram a entrevista estruturada proposta por eles para aplicação das duas últimas escalas. Ainda os mesmos autores, no ano de 1998, apresentaram publicação mostrando esse mesmo valor para o coeficiente de correlação de Spearman, quando somente a DRS e a ERG original foram estudadas ${ }^{(25)}$.

Correlações estatisticamente significativas entre a DRS e a ERG original, com coeficientes variando de 0,50 a 0,98 , também foram identificadas por outros autores ${ }^{(8-9,12)}$.

Segundo resultados observados na Figura 1, a pontuação 3 da DRS foi o ponto de corte importante para diferenciar vítimas dependentes das independentes, uma vez que separou indivíduos classificados com incapacidade grave, na ERG original, dos demais. Análises anteriormente realizadas já apontaram que os escores 0 (zero), 1 ou 2 na DRS definem a condição atual de vida independente ou independente com modificações ${ }^{(27)}$.

Na Figura 2 observa-se que todas as vítimas com recuperação total pela ERG ampliada apresentaram pontuação zero, ou seja, nenhuma incapacidade segundo a DRS, indicando que esta última escala não detectou vítimas com alteração que a ERG ampliada não apontasse. O inverso, porém, não foi observado. Vítimas com leves deficiências (boa recuperação) e até incapacidade moderada na ERG ampliada apresentaram pela DRS indicação de ausência de incapacidades.

Outras pesquisas também têm apontado essa relativa insensibilidade da DRS nas menores pontuações da escala e sua inabilidade para refletir as mais sutis alterações apresentadas pelas vítimas após o trauma ${ }^{(26-30)}$.

Tendo em vista a ERG original e a DRS, alguns estudos que compararam as duas escalas revelaram que a DRS é muito mais sensível para registrar o progresso da vítima póstrauma uma vez que detectou, ao longo do tempo, pequenas mudanças nas condições de vítimas internadas em unidades de reabilitação, que não foram detectadas pela ERG original $^{(6,8)}$.

Outros autores ${ }^{(11)}$ contrapõem-se a esses resultados, mostrando, por meio do coeficiente de variação, maior sensibilidade da ERG original em relação à DRS. Entretanto, a escolha desse coeficiente para essa análise tem sido considerada imprópria, sobretudo quando o caráter ordinal da ERG original é considerado ${ }^{(12)}$.

No presente estudo, a ERG ampliada destacou-se entre as demais escalas, todavia, as características do grupo analisado, provavelmente, favoreceram tais resultados. Observações semelhantes podem ser feitas aos estudos que apontaram a superioridade da DRS sobre a ERG original, realizadas em vítimas internadas em serviços de reabilitação, o que parece trazer algum viés nas conclusões apresentadas.

A superioridade da DRS sobre a ERG original para detectar alterações pós-traumáticas, apontada na análise quantitativa da Tabela 3 é contraposta na Figura 1, quando se observa no grupo com incapacidade moderada, segundo a 
ERG original, pontuação zero na DRS. De forma bem mais freqüente, do que na atual pesquisa, essa incongruência entre as duas escalas foi uma importante observação em estudo realizado por outros autores ${ }^{(7)}$.

A ERG ampliada desde sua concepção traz a premissa de maior sensibilidade do que a ERG original. Avaliações anteriores realizadas em estudo de seguimento de vítimas de TCE, entre seis meses e um ano, mostraram por meio de observação clínica e dos resultados obtidos com o uso das duas versões da escala que, a ERG ampliada torna aparente as mudanças expressivas, tanto em quantidade como em termos de qualidade de vida do indivíduo, deixando, assim, dúvidas se realmente podem ser ignoradas quando as conseqüências pós-traumáticas são analisadas ${ }^{(5,14)}$.

No presente estudo, a DRS embora tenha apresentado uma forte correlação com as duas versões da ERG, mostrouse nitidamente pouco sensível para detectar alterações póstraumáticas, quando comparada à ERG ampliada. Os resultados observados deram indícios de que a DRS pode ser de especial valor para mostrar o progresso de vítimas que se tornaram gravemente incapacitadas pós-trauma, mas é menos adequada na mensuração das conseqüências pós-traumáticas menos graves.

\section{REFERÊNCIAS}

(1) Brasil. Ministério da Saúde. Informações de Saúde. Departamento de Informação e Informática do SUS - DATASUS [online]. Brasília; [s.d.]. Disponível em: http:// tabnet.datasus.gov.br/tabnet/tabnet.html [23 jul. 2004].

(2) National Institutes Health. Consensus Development Panel on Rehabilitation of Persons with Traumatic Brain Injury. JAMA. 1999;282(10):974-83.

(3) Clifton GL, Hayes RL, Levin HS, Michel ME, Choi SC. Outcome measures for clinical trials involving traumatically brain-injured patients: report of a conference. Neurosurgery. 1992;31(5):975-8.

(4) Jennett B, Bond M. Assessment of outcome after severe brain damage. Lancet. 1975;1(7905):480-4.

(5) Jennett B, Snoek J, Bond MR, Brooks N. Disability after severe head injury: observations on the use of the Glasgow Outcome Scale. J Neurol Neurosurg Psychiatry. 1981; 44(4):285-93.

(6) Rappaport M, Hall KM, Hopkins K, Belleza T, Cope DN. Disability rating scale for severe head trauma: coma to community. Arch Phys Med Rehabil. 1982;63(3):118-23.

(7) Pettigrew LE, Wilson JT, Teasdele GM. Assessing disability after head injury: improved use of the Glasgow Outcome Scale. J Neurosurg. 1998;89(6):939-43.
Levando-se em conta tais resultados e a clara indicação da literatura, de que a grande maioria dos sobreviventes de TCE, mesmo que vítimas de trauma graves, apresentam aos seis meses pós-trauma vida independente, pode-se considerar a ERG ampliada como instrumento de escolha em potencial no estudo das conseqüências do TCE, em geral.

Entretanto, acredita-se que o apropriado uso de qualquer uma dessas três escalas depende de se obter uma clara concepção de seus pontos fortes e limitações. Além disso, deve-se considerar a finalidade da mensuração e seu contexto para que falhas nos resultados não sejam propiciadas e, também, indevidas generalizações das conclusões dos estudos sejam evitadas.

Na verdade, não se pode afirmar a existência de um instrumento padrão ouro para mensurar conseqüências pósTCE. O aprimoramento dessas escalas e da forma como utilizálas parece um caminho a ser trilhado, que deve levar em consideração a ampla experiência que a comunidade científica tem tido, utilizando a ERG original e a DRS. O que se tem observado na literatura são, no entanto, muitas posturas tendenciosas que buscam a defesa de um instrumento contrapondo-se a outro, minimizando, muitas vezes, as limitações das escalas e pouco contribuindo para os critérios e aprimoramentos do uso desses instrumentos.

(8) Hall K, Cope N, Rappaport M. Glasgow Outcome Scale and Disability Rating Scale: comparative usefulness in following recovery in traumatic head injury. Arch Phys Med Rehabil. 1985;66 (1):35-7.

(9) Gouvier WD, Blanton PD, LaPorte KK, Nepomuceno C. Reliability and validity of the disability rating scale and the levels of cognitive functioning scale in monitoring recovery from severe head injury. Arch Phys Med Rehabil. 1987;68(2):94-7.

(10) Clifton GL, Kreutzer JS, Choi SC, Davany CW, Eisenberg HM, Foulkes MA, et al. Relationship between Glasgow Outcome Scale and neuropsychological measures after brain injury. Neurosurgery. 1993;33(1):34-9.

(11) Choi SC, Marmarou A, Bullock R, Nichols JS, Wei X, Pitts LW, et al. Primary end points in phase III clinical trials of severe head trauma: DRS versus GOS. J Neurotrauma. 1998;15 (10):771-6.

(12) Wright J, Bushnik T. The GOS vs. the DRS: two recent papers. [on line]. Bethesda; 1999. Available from: < www.thimbs.org/combi> [Access at 2000 dec. 23].

(13) Teasdele GM, Pettigrew LE, Wilson JT, Murray G, Jennett B. Analyzing outcome of treatment of severe head injury: review and update on advancing the use of the Glasgow Outcome Scale. J Neurotrauma. 1998;15(8):587-96. 
(14) Sousa RMC, Koizumi MS. Recuperação das vítimas de trauma crânioencefálico entre 6 meses e 1 ano. Arq Bras Neurocir. 1998;17(2):72-80.

(15) Sousa RMC, Koizumi MS. Recuperação das vítimas de traumatismo crânio-encefálico no período de 1 ano após o trauma. Rev Esc Enferm USP. 1996;30(3):484-500.

(16) Choi SC, Muizelaar JP, Barnes TY, Marmarou A, Brooks DM, Young HF. Prediction tree for severely head-injured patients. J Neurosurg. 1991;75:251-5.

(17) Jennett B, Teasdele G, Braakman R, Minderhoud J, Heiden J, Kurze T. Prognosis of patients with severe head injury. Neurosurgery. 1979;4(4):283-9.

(18) Spettell CM, Ellis DW, Ross SE, Sandel E, O'Malley KF, Stein SC, et al. Time of rehabilitation admission and severity of trauma: effect on brain injury outcome. Arch Phys Med Rehabil. 1991;72(5):320-5.

(19) Strohmyer LL, Noroian EL, Patterson LM, Carlin BP. Adaptation six months after multiple trauma: a pilot study. $\mathrm{J}$ Neurosci Nurs. 1993;25(1):30-7.

(20) Horne G, Schemitsch E. Assessment of the survivors of major trauma accidents. Aust N Z J Surg. 1989;59(6):465-70.

(21) Wagner AK, Hammond FM, Sasser HC, Wiercisiewski D, Norton HJ. Use of injury severity variables in determining disability and community integration after traumatic brain injury. J Trauma. 2000;49(3):411-9.

(22) National Institute on Disability and Rehabilitation Research. Traumatic brain injury model systems national database: variables in database used to address research and demonstration issues. Detroit; 1997.

(23) Maas AI, Braakman R, Schouten HJ, Minderhould JM, Van Zomeren AH. Agreement between physicians on assessment of outcome following severe head injury. J Neurosurg. $1983 ; 58(3): 321-5$
(24) Organização Mundial de Saúde (OMS). Classificação internacional das deficiências, incapacidades e desvantagens (handicaps): um manual de classificação das conseqüências das doenças. Lisboa; 1989

(25) Wilson JT, Pettigrew LE, Teasdele GM. Structured interviews for the Glasgow Outcome Scale and the Extended Glasgow Outcome Scale: guidelines for their use. J Neurotrauma. 1998;15(8):573-85.

(26) Wilson JT, Pettigrew LE, Teasdele GM. Emotional and cognitive consequences of head injury in relation to the Glasgow Outcome Scale. J Neurol Neurosurg Psychiatry. 2000;69(2):204-9.

(27) Hall KM, High W, Wright J, Kreutzer J, Wood D. Functional measures after traumatic brain injury: ceiling effects of FIM, FIM+ FAM, DRS, and CIQ. J Head Trauma Rehabil. 1996;11(5):27-9.

(28) Hall KM, Hamilton BB, Gordon W, Zasler N. Characteristics and comparisons of functional assessment indices: disability rating scale, functional independence measure and functional assessment measure. J Head Trauma Rehabil. 1993;8(2):6074.

(29) Hall KM, Johnston MV. Outcomes evaluation in TBI Rehabilitation. Part II: measurement tools for a nationwide data system. Arch Phys Med Rehabil. 1994;75(12 spec.):SC10-8.

(30) The Center for Outcome Measurement in Brain Injury (COMBI). Introduction to the DRS [online]. Bethesda; 2002. Available from: $<$ www.thimbs.org/combi $>$ [Access at 2000 dec. 23]. 


\section{ANEXOA}

\section{Disability RTING SCALE (DRS).}

\begin{tabular}{|c|c|c|}
\hline CATEGORIA & ITEM & 11 \\
\hline \multirow{3}{*}{$\begin{array}{c}\text { Despertar } \\
\text { Consciência e } \\
\text { Responsividade }\end{array}$} & Abertura ocular ${ }^{(1)}$ & \\
\hline & Habilidade de comunicação ${ }^{(2)}$ & \\
\hline & Resposta motora $^{(3)}$ & \\
\hline \multirow{3}{*}{$\begin{array}{l}\text { Habilidade cognitiva } \\
\text { para atividades de } \\
\text { Autocuidado }\end{array}$} & Alimentação $^{(4)}$ & \\
\hline & Higiene $^{(4)}$ & \\
\hline & Arrumar-se $^{(4)}$ & \\
\hline Dependência de terceiros & Nível de atividade ${ }^{(5)}$ & \\
\hline \multirow[t]{2}{*}{$\begin{array}{l}\text { Capacidade de Adaptação } \\
\text { Psicossocial }\end{array}$} & Desempenho ocupacional $^{(6)}$ & \\
\hline & TOTAL & \\
\hline
\end{tabular}

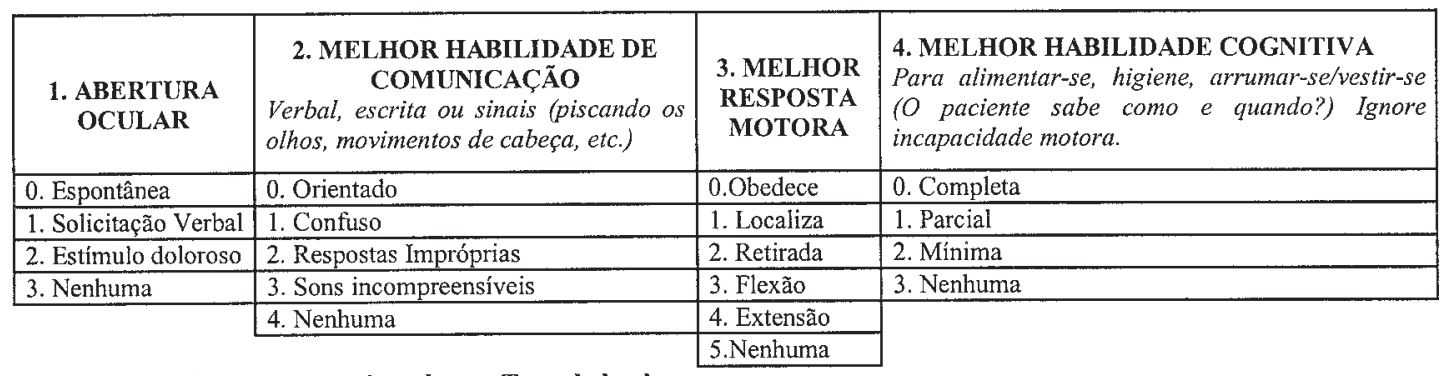

Na presença de traqueostomia coloque $T$ ao lado do escore; para disfuncão verbal ou da fala, coloque $D$ ao lado do escore, (se há disartria, disfonia, paralisia de cordas vocais, afasia, apraxia, etc.)

\begin{tabular}{|c|c|}
\hline $\begin{array}{l}\text { 5. NÍVEL DE ATIVIDADE } \\
\text { Considere tanto incapacidade fisica } \\
\text { quanto cognitiva }\end{array}$ & $\begin{array}{l}\text { 6. DESEMPENHO OCUPACIONAL } \\
\text { Como trabalhador de tempo integral, trabalho doméstico } \\
\text { ou estudante }\end{array}$ \\
\hline 0. Completamente independente & 0. Sem restrição \\
\hline 1. Independente em ambiente especial & 1. Trabalho selecionado/competitivo \\
\hline 2. Levemente dependente $\quad$ (a) & 2. Trabalho adaptado/não competitivo \\
\hline 3. Moderadamente dependente (b) & 3. Incapacitado para ocupações \\
\hline 4. Acentuadamente dependente (c) & \\
\hline 5. Totalmente dependente & \\
\hline
\end{tabular}

(a) Necessita assistência limitada (Cuidador não residente)

(b)Necessita assistência moderada (cuidador em casa)

(C) Necessita assistência em todas

as atividades mais importantes 0

tempo todo

(d) Requer enfermagem $24 \mathrm{~h}$ 


\section{Disability RTING Scale (DRS). Critérios para AplicaÇão}

\begin{tabular}{|c|c|}
\hline $\begin{array}{l}\text { Abertura ocular } \\
0 \text { - ESPONTÂNEA: Olhos abrem com movimento palpebral } \\
\text { normal, indicando a atividade do mecanismo de despertar. Não } \\
\text { considere como indicação de perceptividade. } \\
1 \text { - AO ESTíMULO SONORO OU SENSORIAL: resposta para } \\
\text { qualquer abordagem verbal, não importando o tom de voz, e não } \\
\text { apenas como resposta ao comando de abrir os olhos. Considerar } \\
\text { também a resposta ao toque ou leve pressão. } \\
2 \text { - À DOR: testado com os estímulos dolorosos }{ }^{(1)} \text {. } \\
3 \text { - NENHUMA RESPOSTA: Não há abertura ocular mesmo } \\
\text { com estímulo doloroso. }\end{array}$ & $\begin{array}{l}\text { 1 - PARCIAL: Alterna momentos de consciência, onde sabe } \\
\text { alimentar-se, cuidar-se e higienizar-se ou alterna sua capacidade de } \\
\text { saber quando realizar essas atividades. } \\
2 \text { - MínIMO: mostra questionável ou infreqüente consciência que } \\
\text { sabe de uma maneira primitiva, como comer, cuidar-se e vestir-se, } \\
\text { ou mostra, às vezes, por meio de sinais, sons ou colaboração que é } \\
\text { vagamente capaz de saber quando estas atividades ocorrem. } \\
3 \text { - NENHUM: mostra não estar consciente em qualquer } \\
\text { momento, de que sabe realizar tais atividades por si só. Não } \\
\text { demostra resposta por sinais, sons ou colaboração que mostrem } \\
\text { que ele sabe quando esta atividade pode acontecer. }\end{array}$ \\
\hline $\begin{array}{l}\text { Melhor habilidade de comunicação } \\
\text { (Se o paciente não pode usar a voz devido a traqueostomia, afasia } \\
\text { ou disartria, paralisia das cordas vocais ou com outra disfunção } \\
\text { qualquer da voz, estime qual a melhor resposta do paciente e faça } \\
\text { um comentário a respeito da situação ao final do instrumento.) } \\
\text { 0 - ORIENTADO: demonstra conhecimento de si mesmo e do } \\
\text { ambiente. Está apto a dizer: Quem é? Onde está? Porque está lá? } \\
\text { data (dia, mês, estação e ano) e. hora do dia. } \\
\text { 1 - CONFUSO: a atenção pode ser conseguida e o paciente } \\
\text { responde a questões, mas as respostas são confusas e/ou indicam } \\
\text { variado grau de desorientação e confusão. } \\
2 \text { - RESPOST AS INAPROPRIADAS: palavras compreensiveis, } \\
\text { mas usada somente de forma exclamatória, (como se estivesse } \\
\text { gritando e xingando). Não sustenta comunicação, mudando com } \\
\text { freqüência o assunto ou atenção. } \\
3 \text { - SONS INCOMPREENSÍVEIS: grunhidos, gemidos ou sons } \\
\text { incompreensiveis. } \\
4 \text { - NENHUMA COMUNICAÇÃO: Ausência de resposta } \\
\text { audível ou qualquer comunicação partindo do paciente. }\end{array}$ & $\begin{array}{l}\text { Nível de atividade } \\
0 \text { - COMPLETAMENTE INDEPENDENTE: apto a viver como } \\
\text { desejar, não possuindo restrições físicas, mentais, emocionais ou } \\
\text { sociais. } \\
1 \text { - INDEPENDENTE EM AMBIENTES ESPECIAIS: } \\
\text { capacitado a atividades independentes desde que ajudado por } \\
\text { dispositivos/equipamentos mecânicos auxiliares. } \\
2 \text { - LEVE DEPENDÊNCIA: apto a cuidar da maioria de suas } \\
\text { próprias necessidades, mas requer assistência limitada devido a } \\
\text { problemas emocionais, fisicos ou cognitivos. Ex.: Cuidador não } \\
\text { residente. } \\
3 \text { - MODERADA DEPENDENCIA: apto a cuidar-se } \\
\text { parcialmente mas, precisa de assistência de outra pessoa durante } \\
\text { todo o tempo. } \\
4 \text { - ACENTUADAMENTE DEPENDENTE: necessita ajuda em } \\
\text { todas as principais atividades e assistência de outras pessoas } \\
\text { durante todo o tempo. } \\
5 \text { - TOTALMENTE DEPENDENTE: não está apto a cuidar-se e } \\
\text { requer } 24 \text { horas de cuidados de enfermagem. }\end{array}$ \\
\hline $\begin{array}{l}\text { Melhor resposta motora } \\
\text { 0 - OBEDECE: obedece comando para mover o dedo para seu } \\
\text { lado principal (destro ou sinistro). Se não há resposta ou esta é } \\
\text { inapropriada, tente outro comando como "mover os lábios", } \\
\text { "piscar os olhos", etc. Não solicite aperto de mão ou outras } \\
\text { respostas reflexas, (pegar/agarrar coisas). } \\
\text { 1 - LOCALIZA: o estímulo doloroso }{ }^{(a)} \text { em mais que um local } \\
\text { provoca movimento desta parte, como tentativa de fugir à dor. É } \\
\text { um ato deliberadamente motor de afastamento. Se há dúvida que } \\
\text { tenha sido um movimento de afastamento do estímulo ou em } \\
\text { retirada, teste a resposta ao estímulo doloroso novamente, por } 3 \text { ou } \\
4 \text { vezes. } \\
2 \text { - RETIRADA: qualquer movimento generalizado provocado } \\
\text { por estímulo doloroso. } \\
3 \text { - FLEXÃO: estímulo doloroso que resulta em flexão do } \\
\text { cotovelo, rápida retirada com abdução dos ombros ou leve retirada } \\
\text { com adução dos ombros. Se há dúvida entre flexão e retirada, então } \\
\text { use um objeto pontiagudo sobre a face do paciente. } \\
4 \text { - EXTENSÃO: Estímulo resulta em extensão dos membros. } \\
5 \text { - NENHUMA: Não há resposta visível. Usualmente associada } \\
\text { com hipotonia. Exclua transecção da coluna como causa da } \\
\text { ausência de resposta. Nesse caso, busque uma resposta possível. }\end{array}$ & \multirow{2}{*}{ 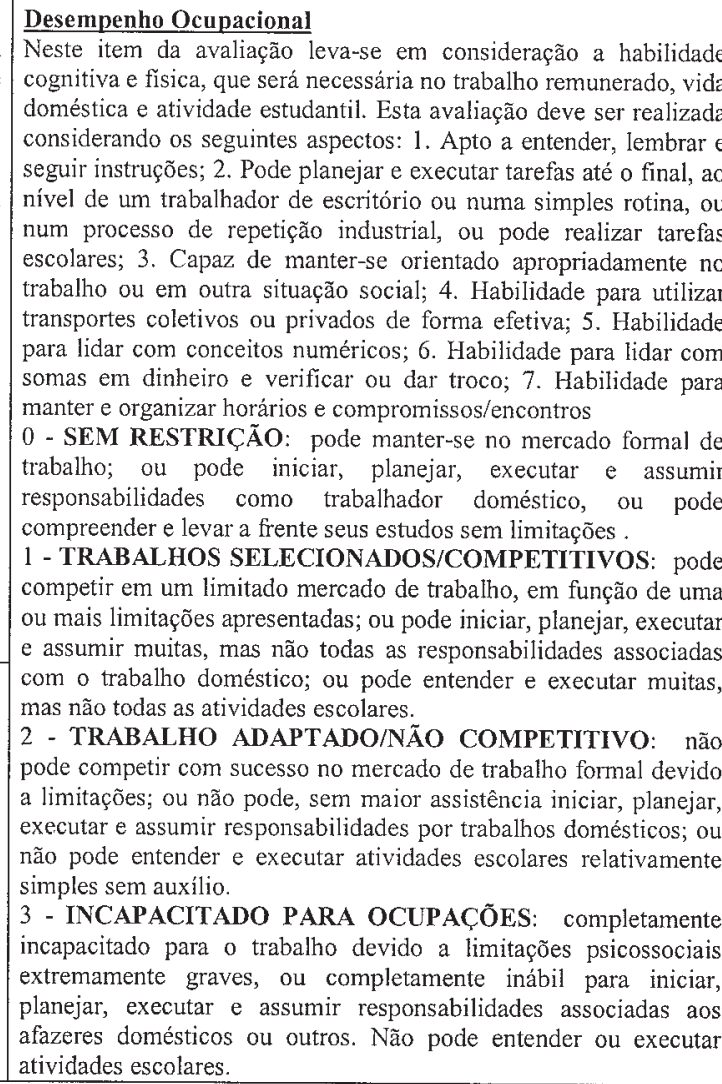 } \\
\hline $\begin{array}{l}\text { Atividades de auto cuidado } \\
\text { (Habilidade cognitiva para alimentar-se, higienizar-se e vestir-se). } \\
\text { Avalia cada uma das } 3 \text { funções separadamente. Para cada função } \\
\text { analise na resposta, se o paciente mostra consciência de como e } \\
\text { quando, deverá realizar cada atividade. Ignore incapacidades } \\
\text { motoras que interfiram na resposta. Avalie a melhor resposta sobre } \\
\text { o cuidado de higiene, baseado no comportamento relativo às } \\
\text { excreções (evacuação e micção). A capacidade de arrumar-se se } \\
\text { refere também ao banho diário, à escovação dentes e cabelo, à } \\
\text { fazer a barba, e por fim vestimenta. } \\
\text { o- COMPLETO: continuamente mostra consciência que sabe } \\
\text { como se alimentar, cuidar-se ou vestir-se sozinho e pode mostrar } \\
\text { claramente que sabe quando esta atividade deverá ocorrer. }\end{array}$ & \\
\hline
\end{tabular}




\section{ANEXO B}

\section{Escala de Resultados de Glasgow ampliada. Critérios para Aplicação}

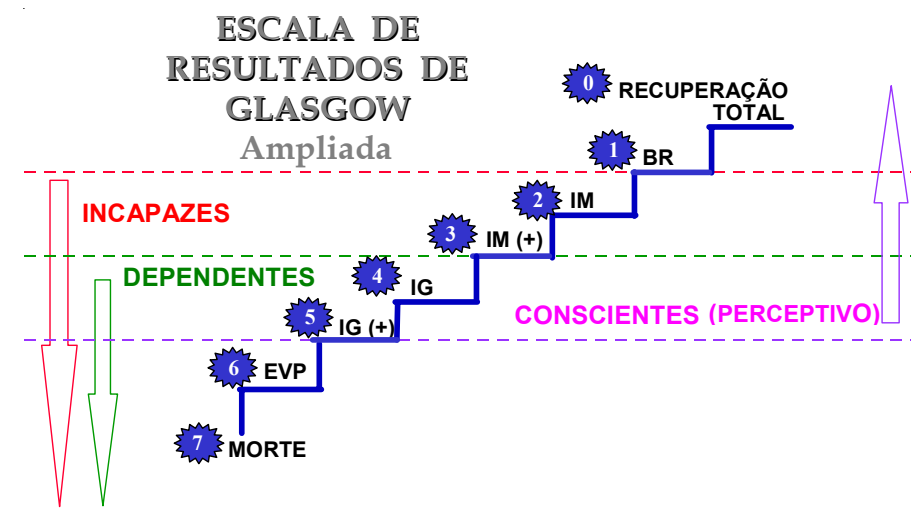

( 0 ) Recuperação total: esta categoria incluí indivíduos que reassumiram vida normal sem nenhuma alteração ou queixa conseqüente ao trauma. Retorno à produtividade não é um parâmetro básico para inclusão nesta categoria.

( 1 ) Boa recuperação (BR): refere-se a indivíduos que reassumiram vida normal com presença de leves deficiências físicas ou mentais. Da mesma forma que na categoria anterior o retorno à produtividade não é parâmetro básico para inclusão na categoria.

( 2 ) Incapacidade moderada (IM): “independentes mas incapazes". Podem realizar as atividades básicas do autocuidado $^{(\mathrm{a})}$ e as outras atividades essenciais para manutenção da independência sem ajuda, por si próprio, com ou sem dificuldades na execução. O ponto básico de diferenciação para boa recuperação é que os indivíduos que são incluídos nessas categorias não conseguem retomar todas as atividades realizadas anteriormente ao trauma.

( 3 ) Incapacidade moderada acentuada $\left(\mathrm{IM}^{+}\right)$: “independente mas incapaz”. Pode realizar as atividades básicas do autocuidado e as atividades essenciais para manutenção da independência, com ajuda de dispositivos ou em meio ambiente em que há modificações para possibilitar sua realização.

( 4 ) Incapacidade grave (IG): “consciente mas dependente”. É capaz de manter as atividades básicas do autocuidado, porém para realizar, uma ou mais, atividades essenciais para manutenção da independência precisa da ajuda de outra pessoa.

( 5 ) Incapacidade grave acentuada $\left(\mathrm{IG}^{+}\right)$: “Consciente mas dependente”. Não consegue manter as atividades básicas do autocuidado sem auxílio de outra pessoa. Necessita de ajuda em pelo menos uma dessas atividades.

( 6 ) Estado vegetativo persistente (EVP): não demonstra evidência de capacidade de elaboração de resposta significativa. Não obedece aos comandos simples e nem pronuncia qualquer palavra. Diferenciar de outras condições em que se tem extrema redução da capacidade de resposta.

( 7 ) Morte: morte em conseqüência ao trauma.

(a) Atividades básicas do autocuidado: banhar-se, alimentar-se, deslocar-se dentro do cômodo/local onde se encontra, manter cuidados referentes a sua função excretora, vestir-se, barbear-se, escovar dentes, pentear-se. 